\title{
Robot-assisted surgery: applications in urology
}

REVIEW

This article was published in the following Dove Press journal:

Open Access Journal of Urology

18 May 2010

Number of times this article has been viewed

\author{
Mathew C Raynor \\ Raj S Pruthi \\ Division of Urologic Surgery, \\ University of North Carolina \\ at Chapel Hill, Chapel Hill, NC, USA
}

\begin{abstract}
The past decade has seen a dramatic shift in the surgical management of certain urologic conditions with the advent of a robotic surgical platform. In fact, the surgical management of prostate cancer has seen the most dramatic shift, with the majority of cases now being performed robotically. Technical refinements over the years have led to improved outcomes regarding oncologic and functional results. Recently, robotic surgery has also been utilized for the surgical management of bladder cancer, renal cancer, and other benign conditions. As further experience is gained and longer-term outcomes are realized, robotic surgery will likely play an increasing role in the surgical management of many urologic conditions.

Keywords: robot-assisted surgery, robotic surgery, cystectomy, prostatectomy, partial nephrectomy
\end{abstract}

Technological advances continue to impact the modern practice of urology, none more so in recent years than the development of robotic surgery. Since the first publication of a series of patients undergoing robot-assisted radical prostatectomy in 2001, the field has seen a dramatic increase in the use of robotic surgery for urologic procedures. In fact, more than 60,000 robot-assisted radical prostatectomies were performed last year alone.

The minimally-invasive nature of these procedures allows for better precision, decreased blood loss, shorter hospital stay, decreased morbidity, and shorter convalescence while preserving functional and oncologic outcomes. Additionally, the application of robotic surgery has spread beyond radical prostatectomy to include radical cystectomy, nephrectomy, partial nephrectomy, adrenalectomy, and other upper urinary tract surgery (pyeloplasty, ureteral reimplant, etc.). Robotic surgery has even seen dramatic growth in pediatric urologic applications.

In this review, we will attempt to highlight robotic surgical applications to various urologic procedures and discuss the recent advances in these procedures.

\section{Robot-assisted radical prostatectomy}

The surgical treatment of prostate cancer has seen a dramatic shift over the past decade. Minimally-invasive approaches to prostate surgery were slow to gain a foothold until the development of a robotic surgical platform. Prior to this development, laparoscopic radical prostatectomy was performed, but only by advanced laparoscopic surgeons. This method was undoubtedly limited by a steep learning curve and the arduous tasks of the procedure, including intracorporeal suturing. The robotic platform takes submit your manuscript | www.dovepress.com

Dovepress

4487
Open Access Journal of Urology 2010:2 85-89

(C) 2010 Raynor and Pruthi, publisher and licensee Dove Medical Press Ltd.This is an Open Access article which permits unrestricted noncommercial use, provided the original work is properly cited. 
advantage of the laparoscopic approach but also allows for more meticulous dissection and ease of suturing due to its greater range of motion and endowrist capabilities.

The steps of robot-assisted radical prostatectomy are similar to those popularized by Menon ${ }^{1}$ and include a transperitoneal approach with a periumbilical camera port $(12 \mathrm{~mm})$ and robotic ports $(8 \mathrm{~mm})$ placed lateral and inferior to the umbilicus. Assistant ports are then placed for suction/ irrigation and passing suture (typically, one $5 \mathrm{~mm}$ port and one $12 \mathrm{~mm}$ port). The space of Retzius is then developed. The endopelvic fascia is then exposed and the periprostatic space is developed distally toward the apex, taking care to preserve the rhabdosphincter. The dorsal venous complex is then ligated. The bladder neck is then dissected and entered anteriorly. The posterior bladder neck is then incised and the ampullae of the vas deferens are approached. Once ligated, the ampullae are used to provide anterior traction while dissection of the seminal vesicles is performed. Denonvillier's fascia is incised and the pre-rectal space is dissected. A nerve-sparing approach is then performed in an antegrade fashion, if clinically indicated, by preserving the lateral prostatic fascia and neurovascular bundles. The procedure then proceeds anteriorly by incising through the dorsal venous complex and urethra. The vesicourethral anastomosis is then performed with a double-armed suture in a running fashion, as initially described by Van Velthoven and colleagues. ${ }^{2}$ Pelvic lymphadenectomy can easily be performed, if indicated, as the initial step in the procedure or prior to the vesicourethral anastomosis.

Since the initial description of the steps of the procedure, there have been multiple technical refinements described in order to maximize oncologic and functional outcomes. Longer term series have shown at least, effective cancer control with the robotic approach as compared with open prostatectomy series. ${ }^{3}$ In a recent review evaluating the outcomes of robot-assisted prostatectomy, pathologic outcomes were similar to open cohorts, with positive surgical margins seen in $15.2 \%$ of specimens. The positive surgical margin rates were found to be $9.6 \%$ and $37.1 \%$ for pathologic stage T2 and T3 prostate cancer, respectively. One single-center comparative study between open and robot-assisted prostatectomy found a decreased rate of positive surgical margins in patients undergoing robot-assisted prostatectomy. This difference was statistically significant for pathologic T2 tumors (9.4\% vs $24.1 \%){ }^{4}$ Perioperative outcomes have also been extensively evaluated. ${ }^{3}$ Intraoperative blood loss is clearly lower with robotassisted or laparoscopic techniques. Overall complication rates are similar between open and robotic approaches. Length of hospital stay varies significantly between studies where most European studies have longer hospital stays than comparative studies from the United States. In general, length of hospital stay is shorter for the robotic approach versus open radical prostatectomy. However, it has been demonstrated that postoperative clinical pathways can be used to shorten hospital stay of open radical prostatectomy equivalent to that of robot-assisted prostatectomy. ${ }^{5}$

Surgical modifications have attempted to improve nerve sparing techniques in an effort to reduce postoperative erectile dysfunction. These modifications include high anterior release of the lateral prostatic fascia ("Veil of Aphrodite", as popularized by Menon and colleagues ${ }^{6}$ ) and techniques to reduce thermal damage to the neurovascular bundles. Descriptions of the use of bulldog clamps to minimize cautery use as well as an endorectal cooling balloon in order to reduce inflammation have been reported in efforts to minimize nerve damage. ${ }^{7,8}$ In addition, an intrafascial nerve sparing procedure may be performed. Recent reports have demonstrated significant short-term improvements in continence and erectile function with the intrafascial approach. ${ }^{9}$ However, the incidence of positive surgical margins is significantly higher for pathologic T3 disease with this method.

Several recent surgical modifications have been aimed at earlier restoration of continence post-operatively. These include reapproximation of the musculofascial plate inferior to the rhabdosphincter to the cut edge of Denonvillier's fascia posteriorly. ${ }^{10}$ This modification stabilizes the vesicourethral junction near the urethral stump and provides a "backboard" support for the bladder neck. Posterior reconstruction has been shown to improve early continence rates, perhaps by restoring membranous urethral length. ${ }^{11}$ Total vesicourethral reconstruction, as described by Tewari and colleagues, involves posterior reconstruction in addition to preservation of the puboprostatic ligaments and the arcus tendineus. ${ }^{12}$ This method of total reconstruction has been shown to improve short-term continence rates, as well. However, other studies have shown no improvement in early continence when comparing single layer anastomosis versus double layer anastomosis incorporating periprostatic tissue. ${ }^{13}$

With recent surgical modifications, earlier improvements in functional outcomes and long-term oncologic efficacy may push robot-assisted radical prostatectomy to the new standard of care for the surgical treatment of prostate cancer. Clearly, robotic surgery has expanded rapidly. However, vigorous debate continues regarding the cost-effectiveness 
of this approach. Development of a robotic surgery program requires significant cost and it is clear that perioperative costs incurred are greater for robot-assisted surgeries. ${ }^{14}$ Minimally-invasive procedures, such as laparoscopic and robot-assisted prostatectomy may lead to decreased blood loss, shorter convalescence, and, arguably, earlier return of erectile function and continence. ${ }^{15}$ It is also evident that there are several advantages to the robotic approach. Only longer-term evaluation of functional and oncologic efficacy, as well as patient satisfaction and quality of life, can be used to justify the increased cost of utilization.

\section{Robot-assisted radical cystectomy}

Robot-assisted radical cystectomy served as a natural progression arising from the advent of robot-assisted radical prostatectomy. Since initial case series reported in 2003, robot-assisted radical cystectomy has become a successful and accepted option in the surgical management of bladder cancer. ${ }^{16}$ Due to the level of complexity of the procedure, robot-assisted radical cystectomy has not attained widespread use and is currently limited to tertiary care referral centers.

A transperitoneal approach is taken with a camera port placed approximately $5 \mathrm{~cm}$ superior to the umbilicus (at least $20 \mathrm{~cm}$ superior to pubic symphysis). Robotic working ports are placed in a similar position to robot-assisted radical prostatectomy, but are shifted 3-4 cm superior to allow for an extended pelvic lymphadenectomy. Initially, extended pelvic lymphadenectomy is performed and is carried proximally at least to the mid-portion of the common iliac vessels. More proximal dissection to the aortic bifurcation and even to the level of inferior mesenteric artery is feasible, if desired. Dissection is carried distally to include the obturator nodal packet and internal iliac packet. Following lymphadenectomy, the ureters are dissected proximally along the psoas and distally to the level of the bladder and transected. The peritoneum in the posterior cul-de-sac is incised and the vasa deferentia are followed proximally toward the prostate. The seminal vesicles are dissected out and incision of Denonvillier's fascia posteriorly allows entry into the prerectal space. Once the posterior dissection is complete, vascular pedicles are developed on each side and ligated down to the level of the endopelvic fascia using an endovascular stapler. At this point, dissection proceeds in an anterior fashion with incision of medial umbilical ligaments and development of the space of Retzius. The endopelvic fascia is then incised and dissected distally toward the apex of the prostate. The dorsal venous complex is suture ligated. A nerve-sparing approach is undertaken, if indicated, with incision of the lateral prostatic fascia. The dorsal venous complex is incised and the urethral stump is mobilized and ligated with a Hem-o-Lok clip (Weck; Teleflex Medical, Research Triangle Park, NC, USA). The Foley catheter can be removed prior to ligating or can be divided after ligation to prevent balloon drainage and tumor spillage. Urinary diversion with either ileal conduit or orthotopic neobladder is then performed extracorporeally after specimen extraction through an infraumbilical incision. Anastomosis of the neobladder to urethral stump can be performed either extracorporeally or intracorporeally with robotic-assistance. Robot-assisted radical cystectomy in the female involves dissection of the posterior cul-de-sac to the level of the vagina. A vaginal-sparing approach is then utilized and the specimen is extracted through a vaginal incision.

With the complexity of the procedure, several surgical modifications have been developed to improve outcomes. Extended pelvic lymphadenectomy can be performed at least as efficiently as open lymphadenectomy with similar lymph node counts and extent of dissection, although this continues to be a point of debate in the urologic oncology community. ${ }^{17}$ Extracorporeal urinary diversion has been shown to be effective in maximizing the benefits of a minimally-invasive procedure and decreasing operating time. Other modifications to improve functional outcomes include prostate-sparing cystectomy in males and uterine-sparing cystectomy in females. Additionally, total intracorporeal urinary diversion is feasible, although with significant increases in operating time.

Recent studies have demonstrated at least equivalent perioperative and pathologic outcomes in robot-assisted cystectomy versus open radical cystectomy. Only short-term and intermediate-term survival analyses have been published, demonstrating at least equivalent survival outcomes for robot-assisted radical cystectomy for bladder cancer when compared to historical open series. ${ }^{18,19}$ One recent randomized trial comparing open to robot-assisted radical cystectomy demonstrated significantly less blood loss, shorter time to flatus, and shorter time to bowel movement with the robotic approach. Length of hospital stay and lymph node counts were not different between the two groups. ${ }^{17}$ As with any robotic procedure, cost is an issue. A recent study showed that robot-assisted cystectomy was associated with higher overall costs compared to open cystectomy. ${ }^{20}$ This difference was mainly due to increased operating room costs for robotic equipment and increased operating room time.

As further refinements in surgical techniques occur and longer-term oncologic and functional efficacy are evaluated, 
robot-assisted radical cystectomy will serve as a viable option for patients undergoing surgical management of bladder cancer.

\section{Robot-assisted partial nephrectomy}

Since the introduction of laparoscopic nephrectomy by Clayman in $1991^{22}$ and the description of laparoscopic partial nephrectomy by McDougall in $1993,{ }^{21}$ minimally-invasive nephron-sparing renal surgery has essentially become the new standard of care for small renal masses. A natural extension of this technology has been the recent expansion of robotic surgery in this field.

Techniques of robot-assisted partial nephrectomy vary in regard to port placement and number, a combined laparoscopic and robotic approach, hilar control techniques, and methods of renorrhaphy. Some advocate an initial laparoscopic dissection to mobilize the colon and expose the renal hilum. Others advocate a completely robot-assisted approach. Regardless of the method, the goal is to expose the renal hilum, enter Gerota's fascia, and identify the renal mass. Typically, a $12 \mathrm{~mm}$ camera port is placed with two $8 \mathrm{~mm}$ robotic arms. Additionally, two assistant ports are placed, usually one $12 \mathrm{~mm}$ port for passage of suture and one $5 \mathrm{~mm}$ port for suction/irrigation. Vascular control of the renal hilum can consist of laparoscopic bulldog or Satinsky clamps. Advantages of the bulldog clamps include their relatively small size, unobstructed view of the kidney, and the ability to isolate individual vascular branches. Disadvantages include the inefficiency of occlusion in some cases and the sometimes cumbersome method of application and removal, which may increase warm ischemia time. Advantages of the Satinsky clamp include the ability to clamp the hilum en bloc with excellent occlusion pressures and to minimize hilar dissection. Disadvantages include the addition of an extra port for placement and the possibility of extracorporeal collision with the robotic arms during hilar clamping. Once the tumor is excised, renorrhaphy is then performed. First, a running absorbable suture is placed in the bed of the defect for hemostasis. Next, sutures are placed in the renal capsule across the defect and Surgicel (Ethicon, Cincinnati, OH, USA) or Gelfoam (Pharmacia and Upjohn, New York, NY, USA) bolsters are placed in the defect. The capsular sutures are then used to secure the bolsters in place and reapproximate renal capsule. Hemostatic agents, such as FloSeal and Tisseel (Baxter Inc., Irvine, CA, USA), are then applied. Gerota's fascia is then reapproximated and the renal mass is extracted. A closed suction drain is left intra-abdominally.
Many different techniques of hilar control during robot-assisted and laparoscopic partial nephrectomy have been described, including bulldog clamps, Satinsky clamps, intra-arterial occlusion catheters, and no clamping at all. ${ }^{23,24}$ Some advocate early unclamping of the hilum after the initial suture is placed in the renal tumor bed. ${ }^{25}$ Others have described methods of obtaining cold ischemia during laparoscopic/robotic procedures. ${ }^{26}$ Despite the method, the goal is an attempt to minimize insult to global renal function.

Due to the rising incidence of small renal masses, robot-assisted renal surgery is likely to play an increasing role in the surgical management of these lesions. In fact, one recent study has even shown a shorter learning curve for robot-assisted partial nephrectomy, thus leading to increased permeation of this surgical modality. ${ }^{27}$

\section{Conclusions}

There has been a dramatic increase in the use of the robotic platform in urologic procedures over the last decade. The majority of robot-assisted procedures include prostatectomy, cystectomy, and partial nephrectomy. Robotic surgery has also been extensively used in the management of ureteropelvic junction obstruction, ureteral strictures, and adrenal surgery. These reconstructive procedures greatly benefit from the added mobility of the robotic instruments and aid in suturing. With continued development of surgical and technological modifications, we are likely to continue seeing increased utilization of robotic surgery for urologic indications. Further analyses regarding cost of a robotic surgical platform and the cost-effectiveness of robotassisted procedures will continue to be explored and debated.

\section{Disclosures}

The authors report no conflicts of interest in this work.

\section{References}

1. Shrivastava A, Baliga M, Menon M. The Vattikuti Institute prostatectomy. BJU Int. 2007;99(5):1173-1189.

2. Van Velthoven RF, Ahlering TE, Peltier A, Skarecky DW, Clayman RV. Technique for laparoscopic running urethrovesical anastomosis:the single knot method. Urology. 2003;61(4):699-702.

3. Coelho RF, Chauhan S, Palmer KJ, Rocco B, Patel MB, Patel VR. Robotic-assisted radical prostatectomy: a review of current outcomes. BJU Int. 2009;104(10):1428-1435.

4. Smith JA, Jr., Chan RC, Chang SS, et al. A comparison of the incidence and location of positive surgical margins in robotic assisted laparoscopic radical prostatectomy and open retropubic radical prostatectomy. J Urol. 2007;178(6):2385-2389; discussion 2389-2390.

5. Nelson B, Kaufman M, Broughton G, et al. Comparison of length of hospital stay between radical retropubic prostatectomy and robotic assisted laparoscopic prostatectomy. J Urol. 2007;177(3):929-931.

6. Menon M, Shrivastava A, Bhandari M, Satyanarayana R, Siva S, Agarwal PK. Vattikuti Institute prostatectomy: technical modifications in 2009. Eur Urol. 2009;56(1):89-96. 
7. Haber GP, Aron M, Ukimura O, Gill IS. Energy-free nerve-sparing laparoscopic radical prostatectomy: the bulldog technique. BJU Int. 2008;102(11):1766-1769.

8. Finley DS, Osann K, Skarecky D, Ahlering TE. Hypothermic nervesparing radical prostatectomy: rationale, feasibility, and effect on early continence. Urology. 2009;73(4):691-696.

9. Potdevin L, Ercolani M, Jeong J, Kim IY. Functional and oncologic outcomes comparing interfascial and intrafascial nerve sparing in robot-assisted laparoscopic radical prostatectomies. J Endourol. 2009;23(9):1479-1484.

10. Rocco F, Carmignani L, Acquati P, et al. Restoration of posterior aspect of rhabdosphincter shortens continence time after radical retropubic prostatectomy. J Urol. 2006;175(6):2201-2206.

11. Nguyen MM, Kamoi K, Stein RJ, et al. Early continence outcomes of posterior musculofascial plate reconstruction during robotic and laparoscopic prostatectomy. BJU Int. 2008;101(9):1135-1139.

12. Tewari A, Jhaveri J, Rao S, et al. Total reconstruction of the vesicourethral junction. BJU Int. 2008;101(7):871-877.

13. Menon M, Muhletaler F, Campos M, Peabody JO. Assessment of early continence after reconstruction of the periprostatic tissues in patients undergoing computer assisted (robotic) prostatectomy: results of a 2 group parallel randomized controlled trial. J Urol. 2008;180(3): 1018-1023.

14. Bolenz C, Gupta A, Hotze T, et al. Cost comparison of robotic, laparoscopic, and open radical prostatectomy for prostate cancer. Eur Urol. 2010;57(3):453-458.

15. Frota R, Turna B, Barros R, Gill IS. Comparison of radical prostatectomy techniques: open, laparoscopic and robotic assisted. Int Braz J Urol. 2008;34(3):259-268; discussion 268-259.

16. Menon M, Hemal AK, Tewari A, et al. Nerve-sparing robot-assisted radical cystoprostatectomy and urinary diversion. BJU Int. 2003;92(3): 232-236.
17. Nix J, Smith A, Kurpad R, Nielsen ME, Wallen EM, Pruthi RS Prospective randomized controlled trial of robotic versus open radical cystectomy for bladder cancer: perioperative and pathologic results. Eur Urol. 2010;57(2):196-201.

18. Martin AD, Nunez RN, Pacelli A, et al. Robot-assisted radical cystectomy: intermediate survival results at a mean follow-up of 25 months. BJU Int. 2009; Nov 9 [Epub ahead of print].

19. Pruthi RS, Wallen EM. Is robotic radical cystectomy an appropriate treatment for bladder cancer? Short-term oncologic and clinical follow-up in 50 consecutive patients. Urology. 2008;72(3):617-620; discussion 620-612.

20. Smith A, Kurpad R, Lal A, Nielsen M, Wallen EM, Pruthi RS. cost analysis of robotic versus open radical cystectomy for bladder cancer. J Urol. 2010;183(2):505-509.

21. Clayman RV, Kavoussi LR, Soper NJ, et al. Laparoscopic nephrectomy. N Engl J Med. 1991;324(19):1370-1371.

22. McDougall EM, Clayman RV, Chandhoke PS, et al. Laparoscopic partial nephrectomy in the pig model. J Urol. 1993;149(6):1633-1636.

23. Boris R, Proano M, Linehan WM, Pinto PA, Bratslavsky G. Initial experience with robot assisted partial nephrectomy for multiple renal masses. J Urol. 2009;182(4):1280-1286.

24. Shen HL, Chueh SC, Lai MK, et al. Balloon occlusion and hypothermic perfusion of the renal artery in laparoscopic partial nephectomy. Int J Urol. 2008;15(11):967-970.

25. Nguyen MM, Gill IS. Halving ischemia time during laparoscopic partial nephrectomy. J Urol. 2008;179(2):627-632; discussion 632.

26. Martins AC, Cologna AJ, Dias-Neto JA, Tucci-Junior S, Suaid HJ. Renal hypothermia: experience in pigs and clinical trial. J Endourol. 2008;22(1):61-64.

27. Deane LA, Lee HJ, Box GN, et al. Robotic versus standard laparoscopic partial/wedge nephrectomy: a comparison of intraoperative and perioperative results from a single institution. J Endourol 2008;22(5):947-952.
Open Access Journal of Urology

\section{Publish your work in this journal}

The Open Access Journal of Urology is an international, peer-reviewed, open access journal publishing original research, reports, editorials, reviews and commentaries on all aspects of adult and pediatric urology in the clinic and laboratory including the following topics: Pathology, pathophysiology of urological disease; Investigation and treatment of

\section{Dovepress}

urological disease; Pharmacology of drugs used for the treatment of urological disease. The manuscript management system is completely online and includes a very quick and fair peer-review system, which is all easy to use. Visit http://www.dovepress.com/testimonials.php to read real quotes from published authors. 